\title{
Centriole Mediated Neurogenesis of OSN in Fish
}

\author{
Subrata Kumar De ${ }^{1}$ and Swaraj Kumar Sarkar ${ }^{1}$ \\ 1. Ultrastructure and Fish Biology Research Unit, Department of Zoology, Vidyasagar University, \\ Midnapore (West) - 721102, West Bengal, India
}

Centrioles are most primitive type of subcellular organelle that is evolutionarily conserved across the phyla [1]. The olfactory sensory receptor neurons (OSN) are primarily equipped with sensory cilia that helps in recognition of chemical cues from the external environment [2]. This type of neuron in fish is uniquely differentiated throughout the life span [3]. This ultrastructural observation simply addresses the question that 'how centriolar morphs influences the olfactory neurogenesis in fish?'

Pseudapocryptes lanceolatus (Bloch and Schneider): a teleostean: Gobiid (common mudskipper) possess unilamellar olfactory apparatus. The olfactory apparatus of adult $P$. lanceolatus was fixed in $2.5 \%$ glutaraldehyde in $0.1(\mathrm{M})$ phosphate buffer $(\mathrm{pH} .7 .2)$ at $4{ }^{\circ} \mathrm{C}$ for 2 hours (primary fixation) and $1 \%$ osmium tetraoxide in the same buffer for 1 hour (secondary fixation). The ultrathin sections (70 90) $\mathrm{nm}$. were stained with uranyl acetate and lead citrate, examined under transmission electron microscope [TEM: MORGAGNI -268D] operated at 40kV [SAIF, All India Institute of Medical Sciences (AIIMS), New Delhi]

The differentiating OSNs are present at the middle part of the pseudostratified olfactory neuroepithelium of $P$. lanceolatus (Figs. 1 and 2). This cell shows two distinct populations of centrioles i.e., type- I centrioles (diameter: $0.2 \mu \mathrm{m}$ ) possess amorphous pericentriolar matrix with 'pinwheel' like arrangement of microtubules and diverging projections (Fig. 1). The type-II of centrioles are centrally placed and devoid of prominent diverging projections and central doublets (Fig. 2). The developmental stage of cilia is associated with type - I centrioles (Fig. 3). The mature olfactory knob of ciliated OSN shows 4 to 6 number of elongated cilia having (9+0) arrangement of microtubules (Fig. 3). Absence of striated rootlets are characteristically recognized in mature olfactory knob of ciliated OSN. The type- I and type- II centrioles are significantly involved in forming cytoskeletal network for vesicular trafficking within the neuroplasm of mature ciliated OSN (Fig. 4).

The kinocilia are originated from type- I centrioles in differentiating OSN whereas the cytoskeletal cross bridges of microtubules are developed from type - II centrioles in mature OSN. The variable centriolar morphs in ciliated OSN are not only responsible for ciliogenesis but also involved in vesicular trafficking for olfactory signal transduction [4] [5].

\section{References:}

[1] MR Mahjoub, Z Xie and T Stearns, The Journal of Cell Biology 191 (2010), p. 331.

[2] S Firestein, Nature 413 (2001), p. 211.

[3] SK Sarkar, TC Nag and SK De, Egyptian Journal of Basic and Applied Sciences 2 (2015), p. 295.

[4] SK De and SK Sarkar, Microscopy and Microanalysis 201420 (2014), p. 1272.

[5] Authors are thankful to Prof. Ranjan Chakrabarti, The Hon'ble Vice Chancellor, Vidyasagar University, India. Prof. S. K. De is also thankful to Department of Science \& Technology, Govt. of West Bengal for partial funding [Memo No. 436 (Sanc.)/ ST/P/S\&T/1G-7/2016 dated 24.08.2016]. 

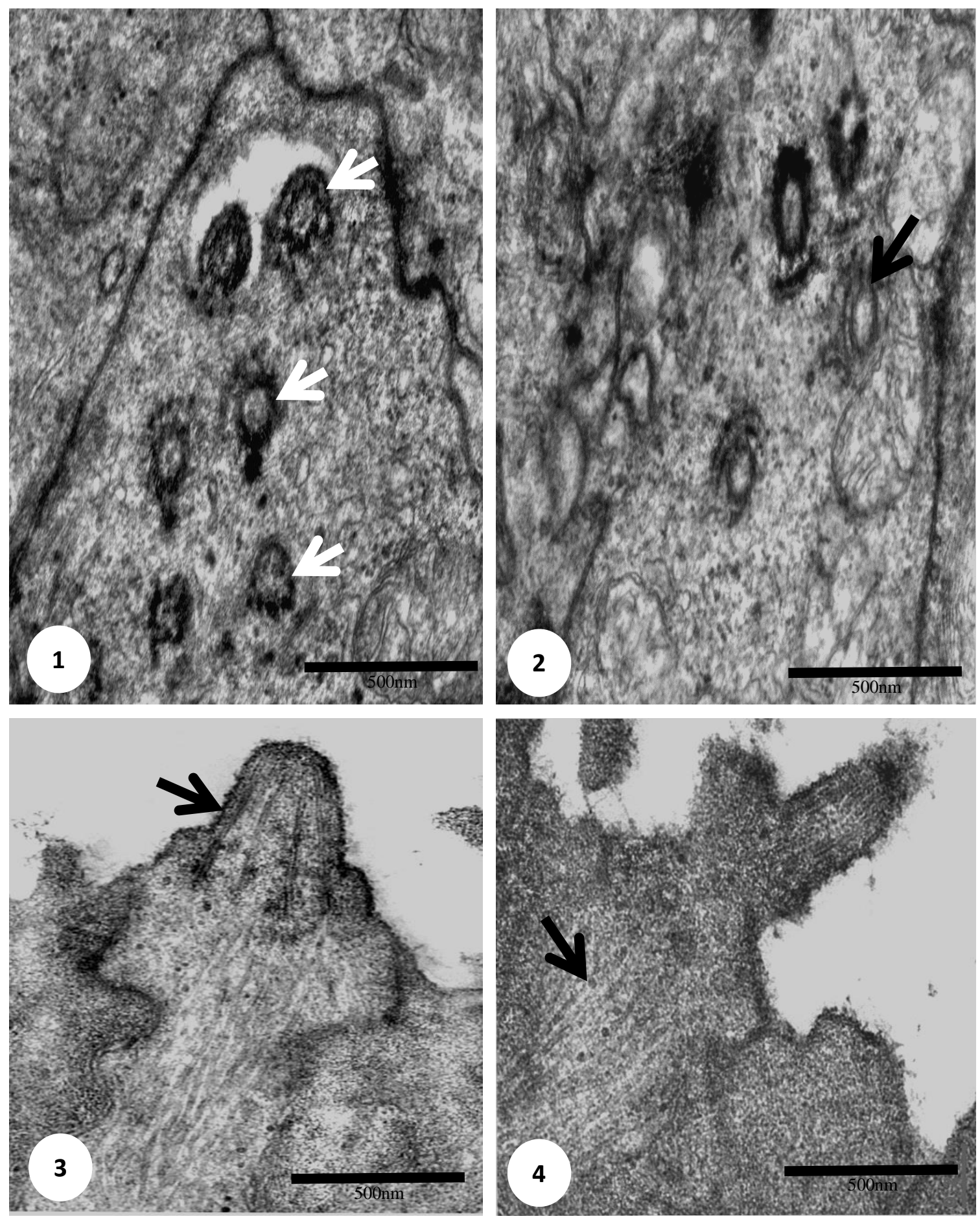

Figure 1. The electron micrograph shows type - I centrioles (arrows) at the proximal cytoplasmic part of differentiating OSN.

Figure 2. The photomicrograph indicates type - II centrioles (arrow) at the central region of differentiating OSN.

Figure 3. The developing cilia associated with microtubules (arrow) are observed under transmission electron microscope (TEM).

Figure 4. The microtubules and small vesicular structure (arrow) are characteristically noted in matured ciliated OSN. 